\title{
Efetividade da reabilitação pulmonar como tratamento coadjuvante da doença pulmonar obstrutiva crônica*
}

\author{
SÉrgio Leite Rodrigues ${ }^{1}$, Carlos Alberto de Assis Viegas ${ }^{2}$, TEREZinha LimA ${ }^{3}$
}

Introdução: O paciente portador de DPOC diminui sua atividade física global devido a piora progressiva da função pulmonar como conseqüência de qualquer forma de esforço físico por ele realizado. A reabilitação pulmonar (RP) é utilizada nos EUA e Europa como alternativa terapêutica no

tratamento da DPOC. Objetivo: Avaliar a efetividade do programa de RP como tratamento

coadjuvante da DPOC. Pacientes e métodos: 30 pacientes prospectivamente submetidos ao programa de RP desenvolvido em seis semanas com freqüência semanal de três sessões. A avaliação compôs-se da história clínica e exame físico completos, do teste de caminhada de seis minutos, do teste de carga máxima sustentada pelos MMSS, do teste de potência máxima de MMII, do questionário

de percepção de esforço físico e espirometria e gasometria. Resultados: No que se refere às variáveis espirométricas e gasométricas pré e pós-programa de RP, não houve alterações estatisticamente significativas $(p>0,05)$. Observaram-se diferenças estatisticamente significativas $(p<$ $0,05)$ nos períodos pré e pós-programa de RP em relação à diminuição da percepção do esforço físico e aumento da capacidade física funcional, do teste de carga máxima para MMSS e testes incrementais de MMII. Conclusões: Para o grupo estudado, os autores concluem que o programa de RP aumentou a sua capacidade física, carga máxima sustentada pelos MMSS e não alterou as variáveis espirométricas

e gasométricas. (J Pneumol 2002;28(2):65-70)

\section{The effectiveness of the pulmonary rehabilitation program as an ancillary treatment for chronic obstructive pulmonary disease}

Introduction: The patient with COPD has his global physical activity decreased due to a progressive worsening of the lung function resultant from any kind of physical effort he may perform. Pulmonary rehabilitation $(P R)$ is used in the United States and Europe as an alternative

therapy in COPD treatment. Objective: To evaluate the effectiveness of $P R$ as an ancillary treatment for COPD. Patients and methods: 30 patients prospectively submitted to the PR program developed in six weeks with three weekly sessions. Evaluation included clinical history and complete physical examination, the six-minute walking distance test, maximum workload test

for upper limbs, maximum load test for lower limbs, a questionnaire of effort perception, spirometry and analysis of blood gases. Results: Concerning spirometry and analysis of blood gases, pre and post $P R$ program, no statistically significant change was observed ( $p>0.05)$. Pre and post PR program, statistically significant values $(p<0.05)$ were observed in the decrease in effort perception and increase in functional physical capacity, maximum workload test for upper

limbs and incremental test for lower limbs used to determine the maximum workload.

Conclusions: The authors concluded that, in the group studied, the PR program increased the patients' physical capacity and maximum workload for upper limbs whereas no changes were observed in the data regarding spirometry and analysis of blood gases.

\footnotetext{
* Trabalho realizado no Serviço de Pneumologia do Hospital Universitário de Brasília, da Faculdade de Medicina da Universidade de Brasília.

1. Mestre em Ciências da Saúde; Especialista em Fisioterapia Respiratória.

2. Doutor em Medicina - Pneumologia.

3. Mestre em Clínica Médica.
}

Endereço para correspondência - Av. Flamboyant, quadra 105, lote 2, apto. 801, Águas Claras - 72030-100 - Taguatinga, DF. Tel. (61) 435-4502 (res.)/933-4616 (cel.); e-mail: sleite99@ig.com.br

J Pneumol 28(2) - mar-abr de 2002

Recebido para publicação em 10/4/01. Aprovado, após revisão, em 29/1/02. 
Descritores - Reabilitação. Condutas terapêuticas. Pneumopatias obstrutivas. Resultado de tratamento. Terapia por exercícios.

Key words - Rehabilitation. Therapeutical approaches. Lung diseases obstructive. Treatment outcome. Exercise therapy.

\section{INTRODUÇÃO}

O paciente portador de doença pulmonar obstrutiva crônica (DPOC) diminui sua atividade física global devido a piora progressiva da função pulmonar, que é traduzida por dispnéia, percepção de cansaço ao realizar qualquer forma de esforço físico. O progressivo descondicionamento físico associado à inatividade dá início a um círculo vicioso, em que a piora da dispnéia se associa a esforços físicos cada vez menores, com grave comprometimento da qualidade de vida ${ }^{(1)}$.

A estratégia utilizada pela reabilitação pulmonar (RP) é integrar-se ao manejo clínico e à manutenção da estabilidade clínica dos portadores de DPOC, principalmente nos pacientes que, mesmo com tratamento clínico otimizado, continuam sintomáticos e com diminuição de sua função física e social ${ }^{(2)}$.

Devido ao comprometimento definitivo da arquitetura pulmonar, gerado pela pneumopatia, a RP não beneficia o paciente no seu quadro de obstrução ao fluxo aéreo. Mas auxilia-o, diminuindo as deficiências e disfunções sistêmicas conseqüentes aos processos secundários da doença pulmonar, como, por exemplo, as disfunções musculares periféricas e respiratórias, anormalidades nutricionais, deficiências cardiovasculares, distúrbios esqueléticos, sensoriais e psicossociais ${ }^{(3)}$.

Dentre os objetivos da RP estão a redução dos sintomas, a redução da perda funcional causada pela doença pulmonar e otimização das atividades físicas e sociais, traduzidas em melhora da qualidade de vida, proporcionando ao paciente a maximização e manutenção da independência funcional. Esses objetivos podem ser alcançados através de processos que incluem o exercício físico, a educação do paciente e de seus familiares e a intervenção psicossocial. A intervenção pela RP visa atender aos problemas e queixas de cada paciente individualmente e é implementada por uma equipe multidisciplinar de profissionais da saúde(4).

Portanto, o principal objetivo deste estudo foi avaliar a efetividade do uso da reabilitação pulmonar como tratamento coadjuvante da DPOC em nosso meio.

\section{PACIENTES}

O estudo descritivo e prospectivo foi realizado com pacientes portadores de DPOC submetidos ao Programa de Reabilitação Pulmonar do Hospital Universitário de
Siglas e abreviaturas utilizadas neste trabalho

CVF - Capacidade vital forçada

DPOC - Doença pulmonar obstrutiva crônica

IMC - Índice de massa corporal

MMII - Membros inferiores

MMSS - Membros superiores

$\mathrm{PaCO}_{2}$ - Pressão parcial de dióxido de carbono no sangue arterial

$\mathrm{PaO}_{2}$ - Pressão parcial de oxigênio no sangue arterial

$\mathrm{pH}$ - O logaritmo negativo, para a base 10 , da concentração de íons livres de hidrogênio em uma solução

RP - Reabilitação pulmonar

$\mathrm{VEF}_{1}$ - Volume expirado forçado no primeiro segundo

$\mathrm{VEF}_{1} / \mathrm{CVF} \%$ - Razão entre volume expiratório forçado e a capacidade vital forçada

VM - Volume minuto

Brasília (HUB) no período compreendido entre julho de 1999 e fevereiro de 2000.

Foram estudados 42 pacientes encaminhados ao Programa de Reabilitação Pulmonar pelo Serviço Ambulatorial de Pneumologia do Hospital de Base do Distrito Federal (HBDF) e do HUB.

\section{Critérios de inclusão}

Foram incluídos os indivíduos encaminhados ao Serviço de Reabilitação Pulmonar do HUB com diagnóstico clínico e funcional de DPOC, com queixas de restrições físicas e sociais devidas a essa patologia, estáveis clinicamente, sem períodos de agudização da doença pelo menos por oito semanas e ex-tabagistas havia, no mínimo, três meses.

Todos os pacientes que participaram do estudo o fizeram de forma voluntária, depois de devidamente esclarecidos e orientados quanto à natureza $e$ ao significado do estudo proposto, assinando um termo de consentimento pós-informado. O protocolo de pesquisa deste trabalho foi aprovado pela Comissão de Ética do Hospital Universitário de Brasília-UnB.

\section{Critérios de exclusão}

Foram excluídos os portadores de doença cardiovascular ou osteoarticular, cuja gravidade os tornava incapazes de realizar seguramente os exercícios apresentados pelo protocolo e os que não estavam estáveis clinicamente ou continuaram com o hábito de fumar.

\section{MÉTODO}

Todos os pacientes selecionados foram submetidos à história clínica e a exame físico completos, realizados pela equipe médica do Serviço de Pneumologia do HUB. Em seguida, foi solicitada a avaliação das variáveis espirométricas e gasométricas, sendo então os pacientes submetidos aos seguintes testes: 
- Teste de caminhada durante seis minutos - consistiu na avaliação da distância máxima percorrida pelo paciente durante seis minutos. Este procedimento foi realizado em uma pista coberta com 25 metros de comprimento. Utilizamos, durante o teste, duas frases padronizadas de incentivo, que foram "O senhor (ou a senhora) está indo muito bem; se for possível, aumente a velocidade da caminhada" no terceiro minuto e "Seu ritmo está ótimo $e$ só falta um minuto para o final; vamos aumentar o ritmo, se for possível" no quinto minuto de caminhada. Inicialmente, cada paciente a percorria duas vezes em dias alternados. A primeira caminhada tinha o propósito de adaptar o paciente ao procedimento adotado e a segunda, de aferir os valores pré-programa. Os parâmetros aferidos antes, durante e após o teste foram a freqüência respiratória, freqüência cardíaca, a saturação da hemoglobina pelo oxigênio $\left(\mathrm{SpO}_{2}\right)$ e a percepção da intensidade de esforço físico pela escala de Borg. Para a utilização da escala, adotamos empiricamente um ponto de corte de 4 (algo grave), visando aferir se a percepção do esforço físico se reduziria a nível moderado ou leve.

- Teste de carga máxima para os membros superiores - consistiu na elevação de pesos realizando um movimento em diagonal com o membro dominante durante dois minutos alternados, com período de repouso de tempo idêntico e com cargas progressivamente maiores $(0,5 \mathrm{~kg})$ até o limite do paciente. O limite foi determinado pela incapacidade de realização do movimento de forma coordenada ou pela incapacidade física de finalizar a seqüência iniciada no tempo programado, sendo esta incapacidade referida pelo paciente.

- Teste de capacidade física máxima - consistiu em pedalar (bicicletas ergométricas) durante oito minutos a uma velocidade entre 18 e $20 \mathrm{~km} / \mathrm{h}$ (40 a 60rpm), e, a cada minuto, foi acrescida uma carga de 10 watts, estando o final do teste condicionado ao limite físico do paciente, referido por este.

\section{Programa de exercícios físicos}

O programa proposto consistiu de exercícios dinâmi$\cos e$ isotônicos desenvolvidos para diferentes grupos musculares localizados nos membros inferiores e superiores.

As sessões de treinamento foram desenvolvidas durante seis semanas com freqüência de três vezes por semana, no período matutino, tendo uma duração média de $1,5 \mathrm{~h}$ incluído o tempo de repouso. A sessão de treino físico foi dividida em quatro etapas:

- $1^{a}$ etapa: exercícios de aquecimento para membros superiores e inferiores, sem carga e com duração de 15 minutos ininterruptos, de acordo com a capacidade de cada paciente, consistindo de atividades calistênicas associadas ao ciclo respiratório;
- $2^{\text {a }}$ etapa: exercícios não-sustentados de membros superiores, utilizando dois movimentos em diagonal com pesos (halteres) durante dois minutos, com intervalo de tempo idêntico para repouso. $\mathrm{O}$ aumento semanal da carga estava condicionado à capacidade individual de cada paciente. $\mathrm{O}$ peso inicial a ser utilizado foi determinado prévia e individualmente pelo teste incremental, com o intuito de trabalhar inicialmente com $50 \%$ da carga máxima sustentada pelo paciente;

- $3^{\mathrm{a}}$ etapa: treinamento de membros inferiores desenvolvido na bicicleta ergométrica com tempo predeterminado e evolução semanal. A potência a ser desenvolvida foi determinada individual e previamente ao início do programa, objetivando um trabalho de $50 \%$ da capacidade física máxima do indivíduo ${ }^{(5,6)}$;

- $4^{\mathrm{a}}$ etapa: posturas de alongamento da musculatura utilizada durante a sessão.

\section{Percepção do esforço físico em atividades diá- rias}

A percepção da intensidade do esforço físico desenvolvido em atividades diárias foi avaliada pré e pós-programa de RP pela associação entre a atividade desenvolvida e a escala de Borg. De forma semelhante ao teste de caminhada, adotamos um ponto de corte de 3 (esforço moderado) na escala de Borg, visando aferir se a redução na percepção esforço físico atingiria nível leve ou muito leve. Utilizamos nove atividades diárias, quais sejam: comer, tomar banho, enxugar-se, pentear-se, barbear-se ou maquiar-se, vestir-se, calçar sapatos ou tênis, subir um andar de escadas (com 12 degraus) e caminhar em terreno plano com passo próprio. Se alguma atividade não se relacionava com a rotina diária do paciente, esta não era avaliada.

\section{Programa educacional}

As orientações educacionais foram ministradas a tais pacientes em seis aulas individuais, durante as seis semanas de programa, e sempre antes do início do treinamento físico. O conteúdo das aulas abordou temas como anatomia e fisiopatologia do sistema respiratório, uso de medicamentos, utilização de oxigênio $e$ a importância do exercício físico na patologia respiratória crônica. As aulas foram ministradas pelo fisioterapeuta e pelo médico assistente.

\section{Análise estatística}

Os dados obtidos são apresentados por estatística descritiva (média e desvio padrão). A comparação entre variáveis numéricas foi feita pelo teste $t$ de Student, e entre variáveis não numéricas, pelo teste do qui-quadrado. Consideram-se estatisticamente significativas as diferenças com $\mathrm{p}<0,05$. 


\section{REsultados}

Foram encaminhados 42 pacientes para o programa de $\mathrm{RP}$, sendo que 12 foram incapazes de participar do grupo: dois tiveram agudização, quatro não tinham condições financeiras para pagar o seu deslocamento até o hospital, um possuía cardiopatia grave e cinco apresentaram problemas osteoarticulares.

A população estudada foi de 30 pacientes, dos quais 27 eram do sexo masculino. A média de idade do grupo era de $66 \pm 8$ anos, variando de 48 a 80 anos. Em relação ao índice de massa corporal (IMC), observamos média de $26 \pm 7 \mathrm{~kg} / \mathrm{m}^{2}$, com valor mínimo de 17 e máximo de $47 \mathrm{~kg} / \mathrm{m}^{2}$.

Na Tabela 1 são apresentados os valores médios, em relação às variáveis espirométricas e gasométricas. Conforme podemos observar, não houve variação estatisticamente significativa $(p>0,05)$ em nenhuma das variáveis estudadas, quando comparados seus valores pré e pósprograma de RP.

No que se refere à aferição da percepção do esforço físico e da capacidade física funcional, utilizamos a escala de Borg durante o teste de caminhada e a distância percorrida durante seis minutos, respectivamente.

Os valores médios evidenciados pela escala de Borg durante os testes de caminhada e a distância total percorrida durante seis minutos dos testes pré e pós-programa de RP podem ser vistos na Tabela 2. Observamos varia-

TABELA 1

Valores espirométricos e gasométricos dos pacientes avaliados pré e pós-programa de RP

\begin{tabular}{lll}
\hline \multicolumn{1}{c}{ Variáveis } & \multicolumn{1}{c}{ Pré } & \multicolumn{1}{c}{ Pós } \\
\hline $\mathrm{PaO}_{2}(\mathrm{mmHg})$ & $67 \pm 12$ & $67 \pm 12^{*}$ \\
$\mathrm{PaCO}_{2}(\mathrm{mmHg})$ & $38 \pm 6$ & $38 \pm 6^{*}$ \\
$\mathrm{SaO}_{2}(\%)$ & $90 \pm 6$ & $92 \pm 5^{*}$ \\
$\mathrm{CVF}^{(\%)}$ & $64 \pm 21$ & $64 \pm 21^{*}$ \\
$\operatorname{VEF}_{1}(\%)$ & $43 \pm 17$ & $42 \pm 16^{*}$ \\
$\operatorname{VEF}_{1} / \mathrm{CVF}(\%)$ & $52 \pm 12$ & $52 \pm 13^{*}$ \\
\hline
\end{tabular}

(\%) pred. = percentual do predito

$\mathrm{mmHg}=$ milímetros de mercúrio

$* p>0,05$

TABELA 2

Valores do escore da escala de Borg e distância total percorrida ao final do teste de caminhada pré e pós-programa de RP, dados apresentados como média \pm desvio padrão

\begin{tabular}{lccc}
\hline & $\begin{array}{c}\text { Pré-RP } \\
(\mathrm{n}=30)\end{array}$ & $\begin{array}{c}\text { Pós-RP } \\
(\mathrm{n}=30)\end{array}$ & $\mathrm{p}$ \\
\hline Escala de Borg & $5 \pm 2$ & $4 \pm 2$ & 0,03 \\
Distância total (metros) & $462 \pm 112$ & $508 \pm 91$ & 0,0003 \\
\hline
\end{tabular}

ção estatisticamente significativa para ambas as variáveis estudadas, escala de Borg e a distância total caminhada durante o teste de seis minutos $(\mathrm{p}<0,05)$. Quando utilizamos o ponto de corte de 4 (algo grave), houve aumento do número de pacientes, 14 pré-RP para 21 pós-RP, classificando o esforço físico, após a caminhada de seis minutos, abaixo do ponto de corte estabelecido.

Em relação aos testes de carga máxima para membros superiores (MMSS) na avaliação pré-programa de RP, obtivemos valor médio de $1,7 \pm 0,5 \mathrm{~kg}$, com valor mínimo de 1 e máximo de $3 \mathrm{~kg}$, que variou para $2,1 \pm 0,5 \mathrm{~kg}$ pósprograma de RP.

Nos testes incrementais de membros inferiores para determinação da potência máxima desenvolvida pré-programa de RP, observamos valor médio de $81 \pm 31$ watts, com valor mínimo de 16 e máximo de 130 watts, e de 93 \pm 33 watts com valor mínimo de 23 e máximo de 156 watts, pós-programa de RP (Tabela 3).

No que se refere à avaliação do nível de dispnéia associado às atividades cotidianas, observamos redução estatisticamente significativa nas atividades de subir um andar de escadas $(p<0,05)$ e vestir-se $(p<0,05)$ quando adotamos um ponto de corte na escala de Borg de 3 (esforço moderado). Em relação a subir um andar de escadas, observamos que apenas sete pacientes classificaram sua dispnéia em nível abaixo do ponto de corte no período préRP em comparação com o período pós-RP, no qual 15 pacientes passaram a perceber a dispnéia abaixo desse nível. Na outra atividade com variação estatística significativa observamos que 12 pacientes apresentavam nível de dispnéia igual ou acima do ponto de corte no período pré-RP. No período pós-RP, apenas cinco pacientes referiram esse nível de dispnéia.

\section{DISCUSSÃO}

Atualmente, um grande número de estudos tem sido realizado com intuito de analisar o valor do programa de RP no tratamento do paciente portador de DPOC. Ao analisar a metanálise de Lacasse et al. ${ }^{(7)}$, podemos observar em 11 ensaios randomizados o aumento da capacidade física máxima em 8,3 watts de potência, avaliada no teste

TABELA 3

Valores individuais e médios dos testes incrementais de carga e potência máximas avaliados pré e pós-programa de reabilitação pulmonar, dados apresentados como média \pm desvio padrão

\begin{tabular}{lccc}
\hline & $\begin{array}{c}\text { Pré-RP } \\
(\mathrm{n}=30)\end{array}$ & $\begin{array}{c}\text { Pós-RP } \\
(\mathrm{n}=30)\end{array}$ & $\mathrm{p}$ \\
\hline Carga máxima para MMSS (kg) & $1,7 \pm 0,5$ & $2,1 \pm 0,5$ & $3,0 \times 10^{-6}$ \\
Potência máxima para M MII (watts) & $81 \pm 31$ & $93 \pm 33$ & $7,0 \times 10^{-6}$ \\
\hline
\end{tabular}


com bicicleta ergométrica, em relação aos controles. Esses trabalhos demonstraram também aumento de 55,7 metros na distância percorrida no teste de caminhada durante seis minutos, no grupo tratado com $\mathrm{RP}$.

Essas informações estimularam-nos quanto à utilização de um programa de RP e posterior avaliação de sua efetividade, no manejo clínico de pacientes portadores de DPOC atendidos no nosso Serviço de Pneumologia, ou seja, em nosso meio, uma vez que não há trabalho brasileiro na referida metanálise.

Relativamente a outras formas de avaliação do treinamento físico regular no paciente portador de DPOC, existem alguns resultados controversos, como o estudo de Belmam et al. ${ }^{(5)}$, que falhou em demonstrar aumento quantitativo de enzimas oxidativas. Entretanto, recente publicação de Maltais et al. ${ }^{\left({ }^{8}\right)}$ conseguiu determinar aumentos significativos de enzimas oxidativas, diminuição do volume minuto (VM) e da produção de lactato nos pacientes submetidos a 12 semanas de treinamento físico.

Para Clark et al. ${ }^{(9)}$, um programa de atividades físicas para portadores de DPOC grave é útil na melhoria da condição física e na melhoria em suas atividades diárias, mesmo adotando níveis baixos de treinamento físico. Nosso estudo apresentou resultados semelhantes, contudo, priorizamos atividades físicas em níveis moderados a máximos. Segundo Casaburi et al. ${ }^{(10)}$, o treinamento físico nesses pacientes pode apresentar resposta fisiológica quando são utilizados níveis próximos da capacidade física máxima.

No presente estudo observamos que houve aumento estatisticamente significativo $(\mathrm{p}<0,05)$ no teste de capacidade física máxima. Com isso, conseguimos um ganho de aproximadamente 12 watts entre o teste inicial e final, concordando com Lacasse et al. ${ }^{(7)}$, que, em sua metanálise sugerem que esse aumento é constante entre os estudos analisados, independente da duração e composição do programa de RP. Para Gosselink e Decramer ${ }^{(11)}$, ganhos na função muscular esquelética podem ser refletidos por melhor rendimento no teste de capacidade física máxima.

No que se refere à avaliação da capacidade funcional, obtivemos resultados significativos e podemos observar ganhos médios de 46,3 metros, indo ao encontros dos resultados obtidos no ensaio randomizado de Bendstrup et al. ${ }^{(12)}$. Sabemos que a distância observada em nosso estudo está entre os valores obtidos em uma metanálise (27,8 a 92,8 metros), em que foram avaliados 14 ensaios randomizados. Os autores consideram que o aumento deve ser de aproximadamente 50 metros para ganhos clínicos importantes no paciente submetido a $\mathrm{RP}^{(7)}$.

Em relação à avaliação da percepção do esforço obtivemos resultados estatísticos significativos ( $p<0,05)$, no sentido de os pacientes referirem menos dispnéia após o teste, a despeito de maior distância percorrida. Entretan- to, nove pacientes $(30 \%)$ falharam em demonstrar melhoria nessa variável, porém, dentre estes, sete aumentaram a distância percorrida durante os seis minutos do teste, o que pode sugerir que a utilização de índices subjetivos pode não se correlacionar de maneira positiva com índices objetivos de aumento da tolerância ao exercício físico.

$\mathrm{Na}$ análise da percepção do esforço físico em atividades cotidianas, encontramos resultados positivos, satisfatórios e estatisticamente significativos apenas nas atividades de vestir-se e subir um andar de escadas $(p<0,05)$. De forma semelhante, em atividades como calçar sapatos ou tênis e caminhar no terreno plano com passo próprio, observamos tendência $(p=0,06)$ de redução na percepção do esforço desenvolvido para estas atividades. Para Garrod et al. ${ }^{(13)}$, a dispnéia pode ser um fator independente do aumento na capacidade de exercício e da melhoria na realização das atividades diárias, aferidos nos testes funcionais.

Em nosso estudo, não observamos alterações estatisticamente significativas em atividades cotidianas que utilizavam os MMSS. Entretanto, no teste de carga máxima, obtivemos aumento nos valores médios de $1,7 \pm 0,5 \mathrm{~kg}$ para $2,1 \pm 0,5 \mathrm{~kg}$ gerando alterações estatisticamente significativas $(\mathrm{p}<0,05)$.

Em relação aos resultados pré e pós-programa de $\mathrm{RP}$ das variáveis de função pulmonar estudadas $\left(\mathrm{pH}, \mathrm{PaCO}_{2}\right.$, $\mathrm{PaO}_{2}, \mathrm{CVF}, \mathrm{FEV}_{1}$ e VEF $/$ /CVF\%), estes não mostraram diferenças estatísticas significativas $(p>0,05)$. Os números refletem que a RP não exerceu nenhuma influência no nível de obstrução das vias aéreas e nos parâmetros gasométricos da população estudada. Esse fato concorda com a literatura pertinente ${ }^{(13)}$, reafirmando o achado de que o programa de RP não influencia as referidas variáveis $e$, sim, os distúrbios secundários à DPOC.

Parece-nos importante reafirmar que a execução dessas atividades cotidianas e necessárias, agora realizadas com menor cansaço, traduz melhoria na autonomia social e física, no sentido de tornar o paciente mais independente, mais ativo fisicamente e, portanto, mais seguro de si mesmo.

Pelo exposto acima podemos concluir que, para o grupo de pacientes estudados, o programa de reabilitação pulmonar aumentou a capacidade física funcional, reduziu a dispnéia durante a realização de atividades cotidianas, aumentou a capacidade física máxima e a carga máxima sustentada pelos membros superiores e, por fim, não alterou as variáveis espirométricas e gasométricas estudadas.

Finalmente, entendemos que o programa de RP trouxe os mesmos benefícios alcançados em outras populações de pacientes portadores de DPOC, devendo, pois, ser considerado efetivo como tratamento coadjuvante dessa enfermidade em nosso meio. 


\section{REFERÊNCIAS}

1. Celli BR. Pulmonary rehabilitation in patients with COPD. Am J Respir Crit Care Med 1995;152:861-4.

2. American Thoracic Society. ATS statement: pulmonary rehabilitation. Am Rev Respir Dis 1981;124:663-6.

3. Lareau SC (Co-chair). Pulmonary rehabilitation-1999: Official statement of American Thoracic Society. Am J Respir Crit Care Med 1999; 159:1666-82.

4. Ries AL. Position paper of the American Association of Cardiovascular and Pulmonary Rehabilitation: scientific basis of pulmonary rehabilitation. J Cardiopulm Rehabil 1990;10:418-41.

5. Belmam MJ, Kendregan BA. Physical training fails to improve ventilatory muscle endurance in patients with chronic obstructive pulmonary disease. Chest 1982;81:440-3.

6. Casaburi R, Wasserman K, Patessio A, Ioli F, Zanaboni S, Donner CF. A new perspective in pulmonary rehabilitation: anaerobic threshold as a discriminant in training. Eur Respir J 1989;2:618s-23s.

7. Lacasse Y, Wong E, Guyatt GH, King D, Cook DJ, Goldstein R. Metaanalyze of respiratory rehabilitation in chronic obstructive pulmonary disease. Lancet 1996;348:1115-9.

8. Maltais F, Leblanc P, Simard C, Jobin J, Berube C, Bruneau J, et al. Skeletal muscle adaptation to endurance training in patients with chronic obstructive pulmonary disease. Am J Respir Crit Care Med 1996; 154:442-7.

9. Clark CJ, Cochrane JE, Mackay E. Low intensity peripheral muscle conditioning improves exercise tolerance and breathlessness in COPD. Eur Respir J 1996;9:2590-6.

10. Casaburi R, Patessio A, Ioli F, Zanaboni S, Donner CF, Wasserman K. Reductions in exercise lactic acidosis and ventilation as a result of exercise training in patients with obstructive lung disease. Am Rev Respir Dis 1991;143:9-18.

11. Gosselink R, Decramer M. Peripheral skeletal muscles and exercise performance in patients with chronic obstructive pulmonary disease. Monaldi Arch Chest Dis 1998;53:419-23.

12. Bendstrup KE, Ingermann Jensen J, Holm S, Bengtsson B. Out-patient rehabilitation improves activities of daily living, quality of life and exercise tolerance in chronic obstructive pulmonary disease. Eur Respir J 1997;10:2801-6.

13. Garrod R, Paul EA, Wedzicha JA. Supplemental oxygen during pulmonary rehabilitation in patients with COPD with hypoxaemia. Tho$\operatorname{rax} 2000 ; 55: 539-43$ 